\title{
Exploiting Collection Level for Improving Assisted Handwritten Word Transcription of Historical Documents
}

\author{
Laurent Guichard - Joseph Chazalon - Bertrand Coüasnon \\ INSA de Rennes, Avenue des Buttes de Coësmes, F-35043 Rennes \\ UMR IRISA, Campus de Beaulieu, F-35042 Rennes \\ Université Européenne de Bretagne, France \\ \{laurent.guichard,joseph.chazalon,bertrand.couasnon\}@irisa.fr
}

\begin{abstract}
Transcription of handwritten words in historical documents is still a difficult task. When processing huge amount of pages, document centered approaches are limited by the trade-off between automatic recognition errors and the tedious aspect of human user annotation work. In this article, we investigate the use of inter page dependencies to overcome those limitations. For this, we propose a new architecture that allows the exploitation of handwritten word redundancies over pages by considering documents from a higher point of view, namely the collection level. The experiments we conducted on handwritten word transcription show promising results in terms of recognition error and human user work reductions.
\end{abstract}

Keywords-document analysis; document sets; handwritten word recognition; historical documents;

\section{INTRODUCTION}

In the context of historical document recognition, transcription of handwritten words is still challenging. Due to the degradation or the specificity of the document handwritings, state-of-the-art automatic recognition is not yet able to fully transcribe this kind of documents. Therefore, human help is necessary to assist the document analysis system; by correcting the detected recognition ambiguities and difficulties.

However, when processing a huge number of document pages, the user annotation work may become tedious.

Even when the system includes a re-training phase to improve over time the recognition, the ground truth for learning requires the human user intervention.

Facing the same limitations of imperfect automatic recognition and of not overloading the human user with annotation, we looked for new and complementary information sources to overcome those restrictions.

As [1] pointed out, the semantic dependencies between document pages could be exploited to improve document recognition. To benefit from this inter pages link, a higher point of view is adopted, namely collection level,

From the collection level, redundancies over pages can be used. For example, some identical words written by the same person contained in two different pages can be grouped together to enforce their individual recognition hypothesis or to be annotated at the same time by a human user. Also, at this level, page contents continuity carries information. For example, the knowledge that handwritten numbers spread over pages form an increasing sequence can be used to optimally recognized them.

For the problem of historical handwritten words transcription, there exists a strong redundancy and homogeneity between document pages. We believe that grouping words at collection level will improve overall document recognition.

To evaluate this idea, we developed a document recognition architecture. In the literature, different kind of architecture are proposed. The DocMining [2] system is similar to a workflow of processing tasks. For each kind of document, a processing scenario is defined, made of tasks such as binarization, connected components extraction, user interaction GUI, etc. The document attached information is updated after each task. While this system is highly configurable, it is document centered and does not provide a common place to manipulate collections of documents. Another system, smartFIX [3], proposes an industrial framework to analysis printed medical bills. It integrates an improving module that can check consistency and optimize interpretations in multi-page documents. This system was aimed at analyzing business documents, hence as they are mainly independent documents, its architecture cannot be configured enough to integrate collection level knowledge.

Keeping the concept of tasks cooperating through a workflow, our architecture has the following specificities to exploit the collection knowledge: It enables tasks to cooperate at collection level through its strategy component; and it manipulates the document information at collection level, thanks to a central storage database component.

By grouping document data at a higher level, our architecture must not break the strong bond between the information at document level and at collection level. Our architecture employs an iterative mechanism to satisfy this constraint.

To summarize, this article has two main contributions :

- present an iterative multi-level architecture to transcript handwritten words;

- demonstrate that processing document at collection level leads to better result in term of user intervention and recognition performances.

This work is organized in the following way. Section II 


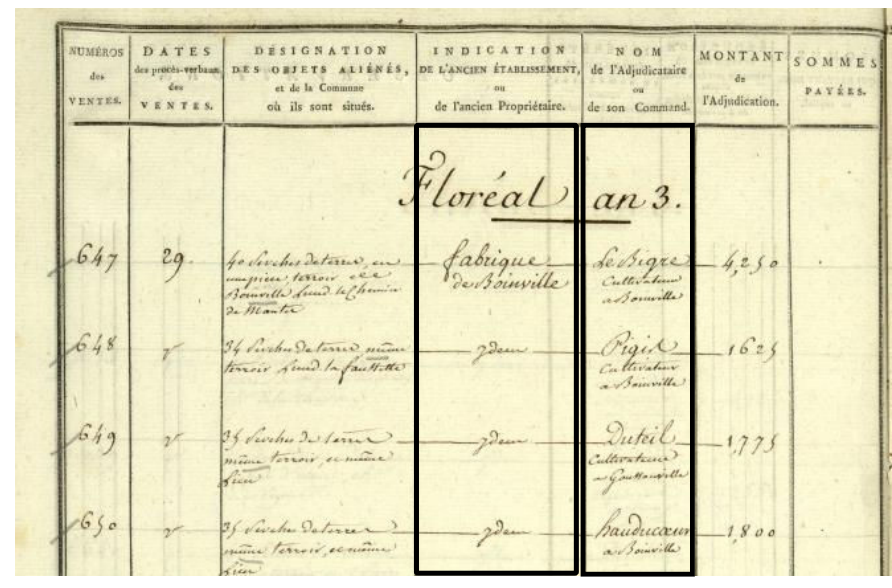

(a)

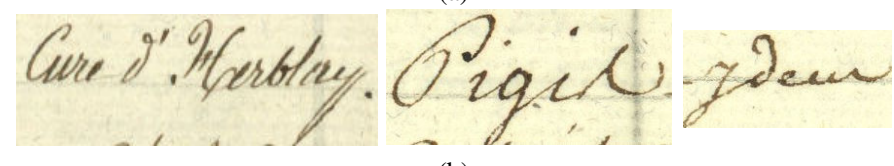

(b)

Figure 1. (a) Sample of the 18th century French revolutionary sales documents to process. Highlighted columns are to be extracted and recognized. (b) Extracted words: "CURE D'HERBLAY', "PIGIS", and "IDEM".

and III address in detail our architecture. Experimental results and conclusion are presented in sections III and IV.

\section{Exploiting Collection Level}

The next two sections detail the strategy and database components needed to work at collection level. Section II-C explains our iterative mechanism to tie together the different levels. Figure 2 synthesizes the different components of our architecture through an example strategy.

\section{A. Cooperation at Collection Level}

Considering the documents from the collection point of view offers new ways of handling their processing. In our case, we are interested in processing the 18th century French revolutionary sales documents, shown in figure 1a, and more precisely in extracting and recognizing the handwritten words in the highlighted columns. Those documents inventory the goods that were sold during the revolutionary sales around year 1791. They are arranged in tables, each row corresponding to a sale. First highlighted column is the former owner and second is the new owner. Some extracted sample words are presented in figure $1 \mathrm{~b}$.

Those documents cannot be recognized automatically because of singular handwriting, time degradation, noise and word overlapping. Thus, human users usually must label by hand some of the words. A simple two stages approach can be used. The first document of the collection is given to a document analyzer which extracts and tries to recognize the handwritten words. Those rejected are annotated by a human user. Then, the second document is processed and so on.
More evolved strategies to handle document recognition could be envisaged. For example, in order to increase the amount of words recognized automatically, we could regroup in clusters all the words amongst the document that graphically "look the same" and then use word individual recognition hypotheses to label the cluster with more confidence. Exploiting word redundancy at collection level by combining word spotting, word clustering and handwritten word recognition could lead to better automatic recognition.

Nevertheless, whatever the ways those processes are assembled, the human user intervention is going to be required. Some of the words would be rejected and they would need to be labeled by hand. But, again, decreasing the amount of work asked to the user could benefit from the collection point of view. Instead of presenting the user one word at a time, he could label the clusters and therefore the annotation of the words in the cluster done in one user action.

Those examples suggest two remarks and their corresponding consequences for our document processing architecture. First, obviously, many king of strategies are possible. Consequently, the presented architecture allows the specification of user own strategies independently from the processing tasks.

Second, the strategy schedules the processing tasks. It is responsible for creating, manipulating and executing the tasks. By considering the documents at collection level, the cooperation of tasks like document analysis, handwritten word clustering and user interaction, is enhanced.

The intra pages regularities can easily be used as information over all documents can be gathered at collection level.

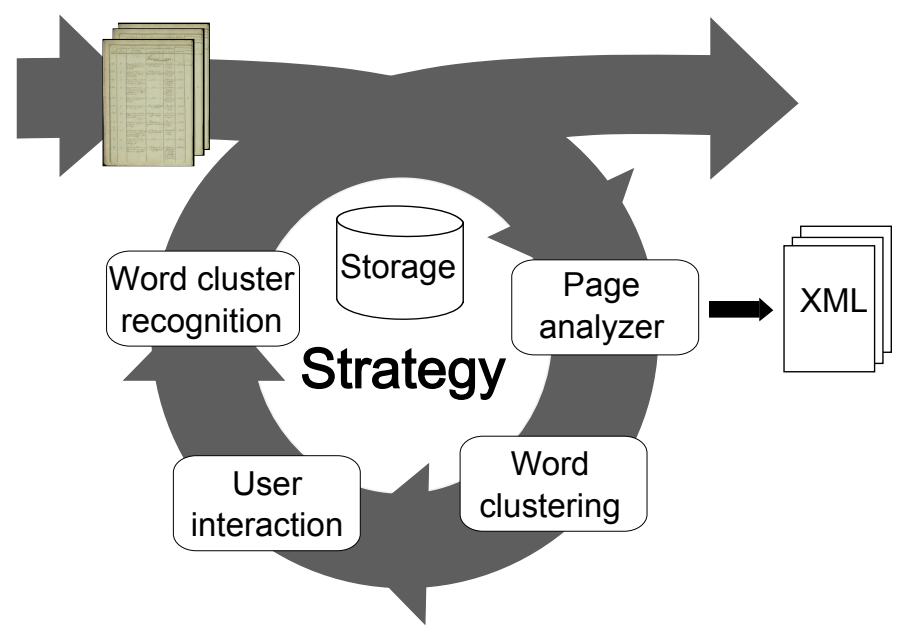

Figure 2. Example strategy to process historical documents based on our iterative architecture

\section{B. Manipulating Document Information}

To efficiently group the redundant handwritten words, the clustering task must be fed with word images extracted from several pages. For this, the strategy is able to collect, 
manipulate and choose the data transiting between tasks so that they work at different levels. The collected data are stored in a central storage database.

Since we are interested in handwritten word recognition, we introduce the main data type field transiting between tasks and stored in the database:

- A contents type

- A 2D bounding box

- A list of top $n$ recognition hypotheses $h_{i}$ defined by a word $w_{i}$ and a confidence score $s_{i}$

- A final word

In the above definition, a word is a transcription of a handwritten word found in a document and that belongs to a known lexicon. The ultimate value of a field is stored in its final word attribute.

Our architecture permits to overcome the difficulty of grouping data extracted from documents. When the strategy, for example, queries the database for all the fields to feed the clustering task, the document physical segmentation is abstracted to consider things at collection level. It implicitly switches from one level to another.

At this point, we have considered only the bottom-up part of our multi level architecture going from document level to collection level. The fields exchanged between tasks are a simple type, they do not embed any higher knowledge about, for example, their semantic connection to another field. It means that some constraints on a field, known only inside the document analyzer task, may not be respected by others tasks that could modify the field. To fulfill the intra document constraints, an iterative scheme is used.

\section{Iterative Mechanism}

As an example of intra-page bond between fields, we can consider the documents illustrated in figure 1a, where the word "IDEM", in the former owner column, means that the name in the current row is the same as the one in the previous row. This knowledge is kept, like any intra-page constraint, in a single page model embedded in the page analyzer to: i) simplify their development; and ii) ease the validation of model constraints in a single component.

When some fields are extracted by the page analyzer but not recognized because of ambiguities, they are stored in the database and another task will take those fields and affects values to their final word attributes that may not take into account their link. Then, the strategy calls again the document analyzer, with, as input, the page and the fields with their actual final word values. The attribute values are kept if no constraint is broken, otherwise they are swept and the whole process is repeated until the document is completely recognized, as symbolized in figure 2 .

The top-down part of the multi level architecture consists in re-injecting those fields into the page analyzer such that the constraints between them is verified, thanks to a method we proposed in [4]. It is not detailed in this paper as we focus on the global architecture enabling the use of collection context. The bottom-up and top-down parts form an iterative mechanism in the multi level architecture, which somehow conciliates the apparent contradiction between centralizing the document knowledge in one task and manipulating the extracted document fields at the collection level.

\section{IMPLEMENTATION OF OUR MULTI LEVEL ARCHITECTURE}

\section{A. Storing Information}

The information to be stored is heterogeneous. Some data are related to the collection level: the list of field clusters. Others are of document level: the fields. To mix them up easily in our database, we chose HBase part of the Hadoop framework ${ }^{1}$. The main advantage of this database is to ease the selection of fields according to the page they belong to, or to their content type: number, family name, city name...

\section{B. Defining the Strategy}

As the Hadoop framework would require some extensions to enable an efficient interaction, we currently use a prototype designed in Python which enables a quick chaining of the various task tools we use. The strategy it implements will automatically run the task processes with appropriate data, and gather their results when their are done, using the database previously presented to store them.

\section{Tasks}

The tasks can be implemented as a $\mathrm{C} / \mathrm{C}++$ shared library, a python script, a binary executable, a remote GUI client, etc. They all respect the same Python interface. We now details the tasks used in the strategies we evaluated in section IV.

1) Page Analyzer: The document recognition is performed using DMOS-P [5], a concept-driven grammatical method for structural analysis of pages, which uses page descriptions to analyze and extract contents. In order to be able to reintegrate manually annotated elements in the document structure and validate them, we use a recent extension of DMOS-P [4] which enables an iterative analysis of document pages. Therefore, according to a page model we defined, a page analyzer processes each page as follows.

- The analyzer is provisioned with all the fields related to the current page stored in the database.

- It locates the textual fields to be transcribed.

- For each field, if a transcription is already available in external data, it is used to fill the final word attribute. Otherwise, the field is submitted to the handwritten word recognition system detailed in [6]. The confidence score associated to the returned word hypothesis is compared to a rejection threshold. If above, the transcription is validated, otherwise it is rejected and marked for external correction.

\footnotetext{
${ }^{1}$ Documentation at http://hadoop.apache.org
} 
- All the fields are sent back to the strategy module.

The incomplete fields may be filled elsewhere in the strategy.

2) Field Clustering: This task aims at regrouping the fields containing the same word. It works on samples which are, in the present case, 2D graphic images corresponding to the field $2 D$ bounding box extracted from the original document image.

First, the samples, transformed to a set of features, are pairwise compared using dynamic time warping to get matching scores that are stored in a cost matrix $M$. As the samples are images containing handwritten text, the extracted features are the one detailed in [7].

Then, a hierarchical agglomerative clustering algorithm is initialized by treating each sample as a cluster. Afterward, the clusters are pairwise merged up till the distance between them exceed a given threshold $T_{c}$. The distance $d_{i, j}$ between clusters $C_{i}$ and $C_{j}$ is computed as:

$$
d_{i, j}=\max _{e_{k} \in C_{i}, e_{l} \in C_{j}} M\left(e_{k}, e_{l}\right)
$$

3) Cluster Recognition: Considering that, at this stage, the clusters newly created are homogeneous, this task goal is to assign to each cluster a word label based on individual field recognition hypotheses.

Let $C$ be a cluster composed of $N$ samples $e_{i}$ and to each sample $e_{i}$ is associated a list of the top $n_{i}$ recognition hypothesis $h_{i, j}, j \in\left[1, n_{i}\right]$ (coming from the field recognition). We define the auxiliary functions: $L: h \mapsto w$ and $S: h \mapsto s$ where $w$ and $s$ are respectively the word and score associated to $h$. Furthermore, we introduce $H_{k}$ as:

$$
H_{k}=\left\{h \mid L(h)=w, w \in W, w \notin H_{i}, \forall i<k\right\}
$$

where $W=\left\{w \mid w=L\left(h_{i, j}\right), i \in[1, N], j \in\left[1, n_{i}\right]\right\}$.

Therefore, a cluster recognition hypothesis $h_{k}$ is defined as a combination of the word $w_{k}=L(h)$ with $h \in H_{k}$ and the following score $s_{k}$ :

$$
s_{k}=\frac{1}{Z} \sum_{\forall h \in H_{k}} S(h) \quad \text { where } Z=\sum_{i=1}^{N} n_{i}
$$

Then, the cluster recognition hypotheses are re-ordered according to their score $s_{k}$, defining a new list: $\left\langle\hat{h}_{1}, \hat{h}_{2}, \ldots\right\rangle$. A thresholding action is performed by assigning $w_{1}=L\left(\hat{h}_{1}\right)$ to cluster $C$, according to:

$$
\text { if } S\left(\hat{h}_{1}\right)-S\left(\hat{h}_{2}\right) \geq T_{r} \text { then accept } w_{1} \text { else reject } w_{1}
$$

where $T_{r}$ is a rejection threshold. The word label assigned to the cluster is spread to the fields constituting the cluster.

It is worth mentioning that the thresholding action relies on the ability of the handwritten word recognizer to efficiently reject ambiguous samples. The recognizer we used was specially developed for this purpose [6].
4) User Interaction: The human user cooperates to the fields recognition by annotating the clusters. The user is presented successively the homogeneous clusters, not automatically recognized by the cluster recognition task. He has a view of one sample of the cluster and he must type the handwritten word he sees. The word label thereby assigned to the cluster is spread to the fields constituting the cluster.

\section{EXPERIMENTS ON ASSISTED HANDWRITTEN WORD TRANSCRIPTION}

The experiments we conducted aim at showing that document processing at collection level can improves both automatic recognition and user annotation work. We present 2 different strategies, one exploiting the collection level. The iterative mechanism is not evaluated here.

\section{A. Test Documents}

For our experiments, 70 document pages looking alike the one in figure 1a where used. It forms a set $S$ of 1206 extracted handwritten fields that need recognition. The localization and extraction were not evaluated in the experiments. Amongst those 1206 fields, they are 502 different words.

\section{B. Tested Strategies}

We compared 2 different strategies made of the tasks detailed in III-C.

Baseline: document pages are processed with the document analyzer, the rejected fields are annotated by a human user depending on threshold $T_{r}$. There is no collection level clustering and no iterative mechanism.

Clustering: document pages are processed with the document analyzer. Clustering task, controlled by $T_{c}$, regroups the fields. Then, the clusters are either automatically recognized or annotated by a human user depending on $T_{r}$.

The number of recognition hypotheses was set to 10 . Both thresholds $T_{c}$ and $T_{r}$ were tuned using a grid search on a validation set.

\section{Experimental setup}

In those experiments, we are focusing on the amount of work for human user. We are evaluating user interaction as described in III-C4.

For the Baseline (resp. Clustering) strategy, a user interaction is to label a field (resp. cluster) by hand.

We aim at minimizing the number of user interactions, in our case, this is equivalent to minimizing the number $N_{M}$ of manual annotations.

This absolute count of manual annotations has to be compared to the worst case where all the fields are labeled by hand, equal to $|S|$.

Thus, we adopt the following definitions for Manual annotation Rate (MR), Error Rate (ER) and Automatic annotation Rate (AR):

$$
M R=\frac{N_{M}}{|S|} \quad E R=\frac{N_{e}}{|S|} \quad A R=1-\frac{N_{M}+N_{e}}{|S|}
$$


Table I

RESULTS FOR TWO STRATEGIES. AR = AUTOMATIC ANNOTATION RATE $(\%)$, MR $=$ MANUAL ANNOTATION RATE $(\%)$, ER $=$ ERROR RATE $(\%)$

\begin{tabular}{|c|c|c|c|c|c|c|c|c|c|}
\hline \multirow{2}{*}{ Strategy } & \multicolumn{3}{|c|}{ W/o reject } & \multicolumn{6}{|c|}{ With reject } \\
\hline & $\mathrm{AR}$ & MR & ER & $\mathrm{AR}$ & MR & ER & AR & MR & ER \\
\hline Baseline & 64 & 0 & 36 & 59 & 21 & 20 & 24 & 75 & 1 \\
\hline Clustering & 66 & 0 & 34 & 63 & 17 & 20 & 38 & 61 & 1 \\
\hline
\end{tabular}

where $N_{e}$ is the number of incorrectly annotated field.

For the Baseline strategy, an incorrectly annotated field is due to an error of the handwritten word recognizer. For the Clustering strategy, an incorrectly annotated field is either caused by a recognizer error or by a clustering mistake. As an example, suppose the clustering has incorrectly regrouped 5 fields in a cluster. 4 have the same label $l$ and 1 has another. If the cluster is assigned automatically label $l$, cluster MR is 0 , ER is 0.2 and AR is 0.8 . If the cluster is assigned manually label $l$, cluster MR is 0.2 , ER is 0.2 and AR is 0.6 .

\section{Results}

Table I presents the results of the experiments for two strategies.

When reject is disabled, there is no manual annotation. A field is either well (goes into AR) or incorrectly recognized (goes into ER). It has to be noted that the recognition of handwritten words in our historical documents is hard as the Baseline strategy only gets $64 \%$ of automatic annotation. In addition, it is worth mentioning that the top $10 \mathrm{AR}$ is of $71 \%(+7 \%)$.

The AR increases from $64 \%$ to $66 \%$ between the Baseline and Clustering strategies. This moderate improvement $(2 \%)$ should be to compared to the top 10 AR of $71 \%$. Indeed, it means that Clustering strategy is able to "recall" $2 \%$ of those potential $7 \%$, i.e. more than $25 \%$ of them.

Reject is used for controlling the error rate. When working in document retrieval domain, the need is to transcribe as many fields as possible with a reasonable amount of manual annotation and error. In this case, the Clustering strategy decreases relatively the amount of manual annotation by $19 \%$, from $21 \%$ to $17 \%$, with an error rate of $20 \%$. The obtained annotation rate (AR + MR) is then $80 \%$.

An error rate of $1 \%$ is appropriate to get automatically some reliable ground truth for recognizer retraining purpose. For such error rate, the Clustering strategy increases relatively the automatic annotation rate by $58 \%$ compared to the Baseline strategy (from 24\% to 38\%). More generally, the Clustering strategy most clearly outperforms the Baseline strategy for low error rate.

\section{CONCLUSION}

This paper introduces a document analysis architecture that allows to process documents at collection level. From this higher point of view, redundancies and homogeneities between pages can be efficiently exploited so as to improve results quality and lower human workload. The architecture is based on three elements: a strategy module; a central database; and an iterative mechanism. The experiments conducted on historical documents show that clustering handwritten words according to their shape leads to an improvement in performances, for two different tasks. For document retrieval which requires the indexing as many elements as possible, a reasonable error rate is conceivable, and the use of collection context permits a relative diminution of $19 \%$ of human workload for an overall annotation rate of $80 \%$. For the adaptation of the system through retraining, a very low error rate is necessary, and our approach enables a relative diminution of $25 \%$ of human workload for an overall annotation rate of $99 \%$.

Our current perspectives are to: i) investigate the impact of the aggregation function which fusion label hypothesis in a cluster, as it could also help recognizing suspicious elements instead of suppressing them; and ii) to quantify the adaptation capability of the system over several passes, after bootstrapping using human-produced ground-truth.

\section{ACKNOWLEDGMENT}

This work has been done in cooperation with the Archives départementales des Yvelines in France, with the support of the Conseil Général des Yvelines.

\section{REFERENCES}

[1] E. Saund, "Scientific challenges underlying production document processing," in Document Recognition and Retrieval XVIII, ser. Proceedings of SPIE, vol. 7874-1, 2011.

[2] E. Clavier, G. Masini, M. Delalandre, M. Rigamonti, K. Tombre, and J. Gardes, "DocMining: A cooperative platform for heterogeneous document interpretation according to user-defined scenarios," in Graphics Recognition, Lladós and Kwon, Eds. Springer, 2004, vol. 3088 of LNCS.

[3] B. Klein, A. Dengel, and A. Fordan, "smartFIX: An adaptive system for document analysis and understanding," in Reading and Learning, Dengel, Junker, and Weisbecker, Eds. Springer, 2004, vol. 2956 of LNCS.

[4] J. Chazalon, B. Coüasnon, and A. Lemaitre, "Iterative Analysis of Pages in Document Collections for Efficient User Interaction," in (also submitted to ICDAR), 2011.

[5] A. Lemaitre, J. Camillerapp, and B. Coüasnon, "Multiresolution cooperation makes easier document structure recognition," International Journal on Document Analysis and Recognition, vol. 11, pp. 97-109, 2008.

[6] L. Guichard, A. Toselli, and B. Coüasnon, "A novel verification system for handwritten words recognition," in International Conference on Pattern Recognition, 2010.

[7] T. M. Rath and R. Manmatha, "Features for word spotting in historical manuscripts," Proc. of ICDAR, vol. 1, p. 218, 2003. 